OPEN ACCESS

Edited by:

Alison Jane Ellen Green, University of Edinburgh,

United Kingdom

Reviewed by:

Thomas Eckland,

Arvinas, Inc., United States

Carlo Scialò,

International School for Advanced

Studies (SISSA), Italy

*Correspondence:

Katsuya Satoh

satoh-prion@nagasaki-u.ac.jp

Received: 01 May 2021 Accepted: 06 September 2021 Published: 28 September 2021

Citation:

Nakagaki T, Nishida N and Satoh K (2021) Development of $\alpha$-Synuclein Real-Time Quaking-Induced Conversion as a Diagnostic Method

for $\alpha$-Synucleinopathies. Front. Aging Neurosci. 13:703984. doi: 10.3389/fnagi.2021.703984

\section{Development of $\alpha$-Synuclein Real-Time Quaking-Induced Conversion as a Diagnostic Method for $\alpha$-Synucleinopathies}

\author{
Takehiro Nakagaki ${ }^{1}$, Noriyuki Nishida ${ }^{1}$ and Katsuya Satoh ${ }^{2 *}$ \\ 1 Department of Molecular Microbiology and Immunology, Nagasaki University Graduate School of Biomedical Sciences, \\ Nagasaki, Japan, ${ }^{2}$ Department of Health Sciences, Unit of Medical and Dental Sciences, Nagasaki University Graduate \\ School of Biomedical Sciences, Nagasaki, Japan
}

Parkinson's disease, dementia with Lewy bodies, and multiple system atrophy are characterized by aggregation of abnormal $\alpha$-synuclein ( $\alpha$-syn) and collectively referred to as $\alpha$-synucleinopathy. Because these diseases have different prognoses and treatments, it is desirable to diagnose them early and accurately. However, it is difficult to accurately diagnose these diseases by clinical symptoms because symptoms such as muscle rigidity, postural dysreflexia, and dementia sometimes overlap among these diseases. The process of conformational conversion and aggregation of $\alpha$-syn has been thought similar to that of abnormal prion proteins that cause prion diseases. In recent years, in vitro conversion methods, such as real-time quaking-induced conversion (RTQulC), have been developed. This method has succeeded in amplifying and detecting trace amounts of abnormal prion proteins in tissues and central spinal fluid of patients by inducing conversion of recombinant prion proteins via shaking. Additionally, it has been used for antemortem diagnosis of prion diseases. Recently, aggregated $\alpha$-syn has also been amplified and detected in patients by applying this method and many clinical studies have examined diagnosis using tissues or cerebral spinal fluid from patients. In this review, we discuss the utility and problems of $\alpha$-syn RT-QulC for antemortem diagnosis of $\alpha$-synucleinopathies.

Keywords: $\alpha$-synuclein ( $\alpha$-syn), Parkinson's disease, dementia with Lewy bodies (DLB), antemortem diagnosis, RT-QuIC, multiple system atrophy (MSA)

\section{INTRODUCTION}

$\alpha$-Synucleinopathies ( $\alpha$-synucleinopathies) are disorders characterized by aggregation and deposition of $\alpha$-synuclein ( $\alpha$-syn), which include Parkinson's disease (PD), dementia with Lewy bodies (DLB), and multiple system atrophy (MSA). PD was first described by British physician James Parkinson in 1817 (Tysnes and Storstein, 2017). PD patients present with motor and autonomic impairments, especially parkinsonisms such as rigidity, rest tremor, bradykinesia, and postural dysreflexia. DLB was first reported by Kosaka (1978) in the late 1970s and is characterized by progressive dementia, visual hallucinations, and parkinsonism (Donaghy and McKeith, 2014); it is the second most common cause of dementia in Japan after Alzheimer's disease (AD). 
Histopathological analysis has revealed neuronal loss and Lewy bodies (LBs) in the cerebrum and brainstem of patients (Spillantini et al., 1997; Baba et al., 1998). Because LBs have been commonly seen in both PD and DLB, these diseases are pathologically named Lewy body diseases (LBDs). However, MSA is a collective term for olivopontocerebellar atrophy, striatonigral degeneration, and Shy-Drager syndrome (Kaji et al., 2020) because of the similarities in symptoms and pathology. Papp et al. (1989) and Nakazato et al. (1990) identified lethargic inclusions in the vacuoles of oligodendroglia in MSA and described the appearance of glial cytoplasmic inclusions (GCIs) in all subtypes of these three diseases. MSA patients present with autonomic symptoms such as dysuria, orthostatic hypotension $(\mathrm{OH})$, and erectile dysfunction, and they are classified as MSA with predominant cerebellar ataxia (MSA-C) and MSA with predominant parkinsonian features (MSA-P) (Gilman et al., 2008; Mitsui et al., 2015).

Because $\alpha$-syn is a major component of $\mathrm{LBs}$ in PD/DLB patients and GCI in MSA patients (Spillantini et al., 1997; Wakabayashi et al., 1998), these diseases are now unified under the concept of $\alpha$-synucleinopathy.

Accurate diagnosis is very important at an early stage of these diseases because deposition of pathological $\alpha$-syn occurs before the symptoms of $\alpha$-synucleinopathies. For example, it has been reported that $50-70 \%$ of dopaminergic neurons are already lost by the time PD is clinically diagnosed (Hughes et al., 1992). Additionally, parkinsonism in DLB has been reported to be less responsive to L-DOPA than that in PD (Lucetti et al., 2010). Thus, even in the same $\alpha$-synucleinopathy, the treatment strategy and prognosis may differ depending on the disease. Moreover, diagnosis of $\alpha$-synucleinopathy by relying on clinical symptoms is difficult because symptoms sometimes overlap between $\alpha$-synucleinopathies and other neurodegenerative disorders including $\mathrm{AD}$, progressive supranuclear palsy (PSP), corticobasal degeneration (CBD), and Creutzfeldt-Jakob disease (CJD) (Armstrong et al., 2013; Hoglinger et al., 2017; McKeith et al., 2017; Geschwind and Murray, 2018; Zerr and Hermann, 2018; Fabbrini et al., 2019). Hence, the most desirable method for preclinical diagnosis is direct detection of aggregated $\alpha$-syn in patients.

In vitro conversion methods, such as protein misfolding cyclic amplification (PMCA) (Saborio et al., 2001; Castilla et al., 2006) and real-time quaking-induced conversion (RT-QuIC) (Wilham et al., 2010), have been developed in the field of prion research. These methods induce conversion of normal prion proteins in brain homogenates or recombinant prion proteins to abnormal prion proteins by sonication or shaking, which allows amplification and detection of trace amounts of abnormal prion proteins in tissues or body fluids. They have been reported by many clinical studies as a preclinical diagnostic method (Hermann et al., 2021).

Aggregated $\alpha$-syn deposition has been reported in various peripheral tissues in patients with $\alpha$-synucleinopathy, including the digestive system, skin, and salivary glands (Beach et al., 2010; Wang et al., 2013; Stokholm et al., 2016; Lee et al., 2017; Niemann et al., 2021), and in CJD patients (Takatsuki et al., 2016; Orru et al., 2017; Satoh et al., 2019). $\alpha$-Syn RT-QuIC using cerebrospinal fluid (CSF), skin, or olfactory mucosa (OM) samples of patients has been developed similarly to prion RTQuIC. Here, we review the most recent findings on $\alpha$-syn RTQuIC using CSF, skin, or OM samples.

\section{$\alpha$-SYNUCLEIN AND REAL-TIME QUAKING-INDUCED CONVERSION}

$\alpha$-Synuclein is a presynaptic protein that consists of 140 amino acids, which can be divided into three domains: N-terminal, middle, and C-terminal domains. The N-terminal domain (aa 1-60) contains the highly conserved repeat KTKEGV and has an $\alpha$-helix propensity. The middle domain (aa 61-95) contains a non-amyloid $\beta$ component (NAC) region and shows a $\beta$ sheet propensity. The C-terminal domain (aa 96-140) is enriched with proline and negatively charged amino acids (glutamine and asparagine) and is an intrinsically disordered region that contributes to the maintenance of solubility (Breydo et al., 2012; Villar-Piqué et al., 2016; Wang et al., 2016). $\alpha$-Syn has been reported to be a soluble and monomeric disordered protein in neurons, but has also been reported to form macromolecular assemblies and adopt a variety of structures. $\alpha$-Syn aggregation is induced by deletion of its C-terminal domain. Hence, the interaction with the NAC region plays a crucial role in its structural stability (Crowther et al., 1998; Bertoncini et al., 2005). Furthermore, $\alpha$-syn undergoes post-translational modifications of which one of the most common is phosphorylation of serine 129 (S129), which is found only in pathological $\alpha$-syn deposits (Fujiwara et al., 2002; Doppler et al., 2014). S129 phosphorylation of recombinant $\alpha$-syn has been reported to accelerate polymerization and promote aggregation (Fujiwara et al., 2002; Sano et al., 2018). It has also been reported that pathological changes are suppressed by inhibiting this phosphorylation in animal models. Thus, S129 phosphorylation is thought to have a significant effect on $\alpha$-syn aggregation.

From another perspective, when nearly $40 \%$ of the volume is occupied by RNA and proteins, the intracellular environment becomes extremely crowded and water activity reduces (Gnutt and Ebbinghaus, 2016; Rivas and Minton, 2016). In this situation, the protein structure becomes compact, which promotes aggregation (Cheng et al., 2018). This condition has been reported to promote aggregation of disease-associated proteins that include $\alpha$-syn, FUS, and PrP (Murray et al., 2017; de Oliveira et al., 2019; Ray et al., 2020; Tange et al., 2021). Disruption of the interaction between the NAC region and C-terminal domain by the environment, mutations, or post-translational modifications has been considered to promote $\alpha$-syn aggregation.

The QuIC assay is capable of detecting a very small amount of abnormal protein added as a seed to reaction buffer that contains a recombinant protein purified from Escherichia coli as the substrate; this is repeatedly and intermittently shaken and then left to stand. Thus, the products of the RT-QuIC reaction have a $\beta$-sheet-dominant structure from the $\alpha$-helix-dominant structure, thereby promoting amyloid formation (Figure 1). Therefore, the structure of the substrates is induced to convert to the abnormal form by the QuIC reaction (Atarashi et al., 2008; 


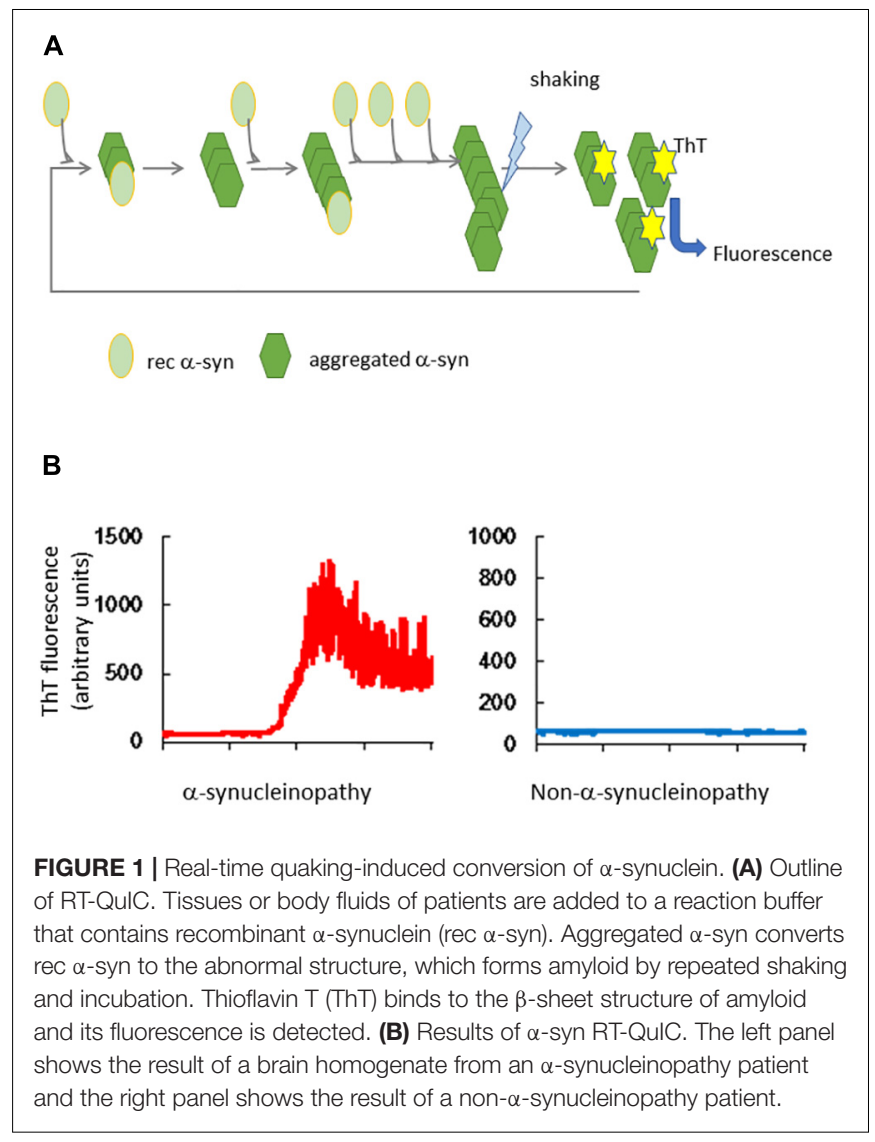

Wilham et al., 2010). Thioflavin T (ThT), which is added to the reaction buffer, binds to amyloid and its fluorescence is measured at certain intervals to observe the amyloid formation reaction in real time (Atarashi et al., 2011).

The most commonly reported diagnostic method uses CSF of human prion patients (Atarashi et al., 2011; Sano et al., 2013; Foutz et al., 2017). Detection of abnormal aggregated $\alpha$-syn in CSF of DLB patients by the RT-QuIC assay was first reported by Fairfoul et al. (2016); this was followed by multiple clinical studies. At present, diagnostic methods using skin (Orru et al., 2017) or OM (Orru et al., 2014; Bongianni et al., 2017), which includes CSF, have been reported (Satoh et al., 2017; Ascari et al., 2020; Candelise et al., 2020).

\section{DETECTION OF AGGREGATED $\alpha$-SYNUCLEIN IN CEREBROSPINAL FLUID}

Lumbar puncture is one of the most commonly performed procedures by neurologists. Therefore, RT-QuIC using spinal fluid has been widely studied as an effective method for early diagnosis of prion diseases (Atarashi et al., 2011; Sano et al., 2013; Foutz et al., 2017). CSF is also considered to be the most promising material for clinical diagnosis by $\alpha$-syn RT-QuIC (Table 1).
In the first report of $\alpha$-syn RT-QuIC, the authors investigated the amount of CSF used as the seed in pure DLB $(n=12)$ patients who were neuropathologically diagnosed in the OPTIMA cohort study compared with normal controls (Fairfoul et al., 2016). The sensitivity and specificity of pure DLB were 83 and $96 \%$, respectively, when the volume of CSF was $5 \mu \mathrm{L}$. The sensitivity was increased up to $92 \%$ with $10 \mu \mathrm{L}$ CSF and $98 \%$ with $15 \mu \mathrm{L}$, and the specificity of $\alpha$-syn RT-QuIC was $100 \%$ when the seeds were both 10 and $15 \mu \mathrm{L}$ CSF from patients clinically diagnosed with $\alpha$-synucleinopathies. CSF obtained from patients with an unclear diagnosis of parkinsonism has been tested by $\alpha$-syn RT-QuIC. The sensitivity and specificity were 75 and 94\%, respectively (Table 1; van Rumund et al., 2019). This is the first report to examine a case in which no clinical diagnosis had been made. The lower sensitivity was thought to be because the test was performed in patients with an unclear diagnosis (only 50\% of patients were diagnosed with $\alpha$-synucleinopathy at 3 years after their lumber puncture). Then, a large cohort study of 439 clinically diagnosed and autopsied cases was performed. The sensitivity of 21 autopsied cases pathologically determined to be positive for LB- $\alpha$-syn was $95.2 \%$ and the specificity in all $101 \mathrm{LB}$ $\alpha$-syn-negative cases was 98\% (Rossi et al., 2020). In the clinical cohort, the sensitivity was $95.4 \%$ and the specificity was 94\% (Table 1).

Kang et al. (2019) compared the diagnostic accuracy of RTQuIC (1 min shake at $200 \mathrm{rpm}$ followed by $14 \mathrm{~min}$ rest) and PMCA in PD (1 min shake at $500 \mathrm{rpm}$ followed by $29 \mathrm{~min}$ rest) patients from the BioFIND cohort and CSF from healthy controls. The sensitivity of RT-QuIC and PMCA in 105 PD patients was 96.2 and $95.2 \%$, respectively, and the specificity of RT-QuIC and PMCA in the 79 healthy controls was 82.3 and $89.9 \%$, respectively. The concordance rate between the two was as high as $92 \%$, which indicated reliability and reproducibility of PD diagnosis.

Groveman et al. (2018) performed RT-QuIC at a higher temperature $\left(30-42^{\circ} \mathrm{C}\right)$, higher shaking speed (200-400 rpm), and shorter incubation period $(14 \rightarrow 1 \mathrm{~min})$ than the original assay and added $0.0015 \%$ SDS to the reaction buffer. They succeeded in shortening the lag phase from 50 to $20 \mathrm{~h}$ (Groveman et al., 2018). Furthermore, using a mutant $\alpha$-syn (K23Q) as the substrate, they suppressed the slight increase in ThT fluorescence observed in healthy control CSF when WT $\alpha$-syn was used as the substrate. The sensitivity of the assay using CSF of 12 PD and 17 DLB patients was 91.6 and $94.1 \%$, respectively, and the specificity in 31 non- $\alpha$-synucleinopathies, which included $16 \mathrm{AD}$ cases, was $100 \%$. They named this method rapid RTQuIC (RT-QuICR) (Groveman et al., 2018). Additionally, they detected aggregated $\alpha$-syn by RT-QuICR in the CSF of PD patients from the same BioFIND cohort as Kang et al. (2019) and compared the accuracy with the original RT-QuIC and PMCA. The specificity of RT-QuICR in 108 PD samples was $97.2 \%$, and in 85 healthy controls, it was $87.1 \%$, which was comparable with the other two assays (Orrù et al., 2020). The sensitivity and specificity of $\alpha$-syn RT-QuIC using CSF for PD and DLB diagnoses have been sufficiently reliable in various studies (Table 1). 
TABLE 1 | Sensitivity and specificity of $\alpha$-syn RT-QulC in CSF of patients

\begin{tabular}{|c|c|c|c|c|c|c|c|c|}
\hline Method & Substrate & $\alpha$-Synucleinopathy & Number & Sensitivity (\%) & Non- $\alpha$-synucleinopathy & Number & Specificity (\%) & References \\
\hline \multirow[t]{27}{*}{1} & WT & Total & 32 & 93.8 & Total & 55 & 100 & Fairfoul et al., 2016 \\
\hline & & $\mathrm{PD}$ & 20 & 95.0 & $A D$ & 30 & 100 & \\
\hline & & DLB & 12 & 91.7 & Healthy & 20 & 100 & \\
\hline & & & & & $\mathrm{CBD}$ & 3 & 100 & \\
\hline & & & & & PSP & 2 & 100 & \\
\hline & WT & Neuropathologically verified cases & & & & & & Bongianni et al., 2019 \\
\hline & & Total ( $\alpha$-synucleinopathies) & 28 & 92.9 & Total (non- $\alpha$-synucleinopathy) & 49 & 95.9 & \\
\hline & & DLB & 7 & 100 & sCJD & 19 & 100 & \\
\hline & & MSA-C & 1 & 100 & Other neurodegenerative diseases & 11 & 90.9 & \\
\hline & & LBD/AD & 15 & 93.3 & & & & \\
\hline & & LBD/PART & 2 & 100 & Other neurological diseases & 19 & 94.7 & \\
\hline & & CJD/LBD & 3 & 66.7 & & & & \\
\hline & & Clinical cases & & & & & & \\
\hline & & Probable DLB & 20 & 85.0 & Probable AD & 10 & 100 & \\
\hline & & Possible DLB & 6 & 0 & & & & \\
\hline & WT & Total & 85 & 75.3 & Total & 78 & 93.6 & van Rumund et al., 2019 \\
\hline & & $\mathrm{PD}$ & 53 & 84.9 & PSP & 8 & 87.5 & \\
\hline & & MSA & 17 & 35.3 & Tauopathy of uncertain origin & 2 & 100 & \\
\hline & & DLB & 1 & 100 & VaP & 9 & 66.7 & \\
\hline & & $\alpha$-Synucleinopathy with vasculopathy & 11 & 81.8 & Other & 7 & 100 & \\
\hline & & & & & Control & 52 & 98.1 & \\
\hline & & $\alpha$-Synucleinopathy of uncertain origin & 3 & 100 & & & & \\
\hline & WT & $\mathrm{PD}$ & 105 & 96.2 & Healthy & 79 & 82.3 & Kang et al., 2019 \\
\hline & WT & Total of LRRK2 mutation & 31 & 29.0 & & & & Garrido et al., 2019 \\
\hline & & LRRK PD & 15 & 40.0 & Healthy control & 10 & 20.0 & \\
\hline & & LRRK NMC & 16 & 18.8 & & & & \\
\hline & & IPD & 10 & 90.0 & & & & \\
\hline \multirow[t]{2}{*}{2} & WT & PD & 15 & 100 & PSP & 5 & 60.0 & Manne et al., 2019 \\
\hline & & & & & No $\alpha$-synucleinopathy & 11 & 100 & \\
\hline \multirow[t]{10}{*}{2} & WT & Total of definite cases & 21 & 95.2 & Total of definite cases & 101 & 98.0 & Rossi et al., 2020 \\
\hline & & DLB & 14 & 100 & $A D$ & 17 & 94.1 & \\
\hline & & Dementia with incidental LB & 7 & 85.7 & PSP & 1 & 100 & \\
\hline & & & & & MSA & 2 & 100 & \\
\hline & & & & & Syn-controls & 81 & 98.8 & \\
\hline & & Total of clinical cases & 151 & 95.4 & Total of clinical cases & 166 & 94.0 & \\
\hline & & DLB & 34 & 97.1 & $A D$ & 43 & 83.7 & \\
\hline & & $\mathrm{PD}$ & 71 & 94.4 & Clinical controls & 62 & 98.4 & \\
\hline & & & & & PSP/CBS & 30 & 100 & \\
\hline & & & & & MSA & 31 & 93.5 & \\
\hline
\end{tabular}




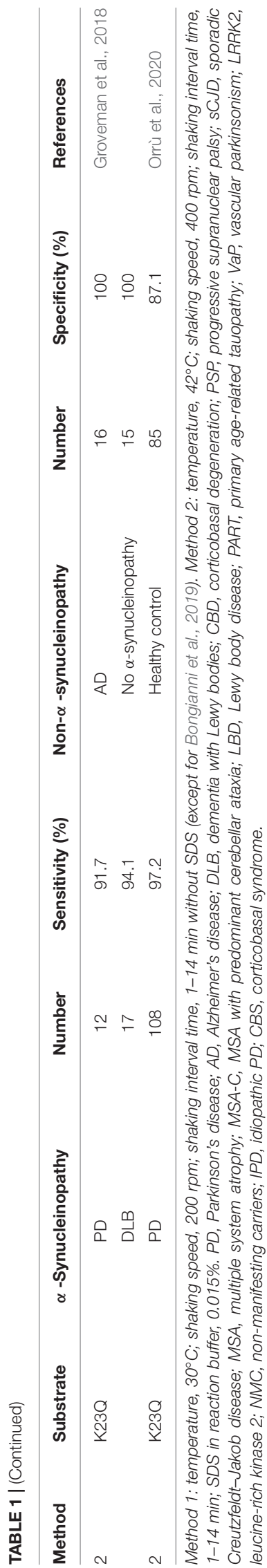

\section{DETECTION OF AGGREGATED $\alpha$-SYNUCLEIN IN FAMILIAL PARKINSON'S DISEASE PATIENTS}

Although all previous studies have focused on idiopathic $\alpha$ synucleinopathy, a study on patients with the p.G2019S mutation in the leucine-rich kinase 2 (LRRK2) gene has also been reported. This mutation is the most frequently found in familial PD (Healy et al., 2008; Mancini et al., 2020). Garrido et al. (2019) tested patients with idiopathic PD (IPD), LRRK2-PD, or LRRK2 nonmanifesting carriers (NMCs). IPD was positive in 9 of 10 patients (sensitivity 90\%), whereas RT-QuIC positivity for LRRK2-PD was found in 6 of 15 patients (40\%) and LRRK-NMC was found in only 3 of 16 patients (18.8\%) (Table 1 ). Two of 10 healthy controls were positive (specificity $80 \%$ ), and unlike IPD patients, LRRK2PD patients had a lower rate of a positive reaction in the RT-QuIC assay, which the authors attributed to less insoluble synuclein and lower seed activity in the brain of LRRK2-PD patients compared with IPD patients. These data indicated that sensitivity for familial PD is lower than that for IPD. Further research is needed to increase the number of cases and to investigate other mutations.

\section{IMMUNOSTAINING ANALYSIS OF $\alpha$-SYNUCLEIN IN SKIN}

Deposition of $\alpha$-syn in dermal nerve fibers and decrease sof autonomic innervations of sweat glands, blood vessels, and erector pili muscles have been reported in PD patients. Wang et al. (2013) successfully detected native $\alpha$-syn using an anti$\alpha$-syn antibody in dermal nerve fibers of PD patients and healthy controls. They also reported that the density of $\alpha$-synpositive nerve fibers in skin tissue was increased in PD patients compared with healthy subjects (Wang et al., 2013). Deposition of the disease-associated form, phosphorylated $\alpha$-syn (p- $\alpha$-syn), in dermal and epidermal fibers has also been reported. Gibbons et al. (2016) detected $\mathrm{p}-\alpha$-syn and quantified deposition of $\alpha$ syn in the pilomotor or sudomotor of skin biopsies and found that $\alpha$-syn deposition was higher in patients without autonomic symptoms than in healthy subjects (the sensitivity and specificity for PD compared with normal subjects was $>90 \%$ ). Other groups have reported that specific p- $\alpha$-syn can be detected in skin biopsies by pathological findings (Ikemura et al., 2008; Donadio et al., 2016). The sensitivity and specificity of anti-phosphorylated synuclein antibodies have been analyzed by meta-analysis of 41 case-control studies that included 12 studies using skin tissues of patients (Tsukita et al., 2019). Three studies (Navarro-Otano et al., 2015; Donadio et al., 2016; Melli et al., 2018) (38 PD patients and 42 controls) using anti- $\alpha$-syn antibodies showed $76 \%$ sensitivity and $60 \%$ specificity. In nine studies using anti-p- $\alpha$-syn antibodies (170 PD patients and 214 controls) (Donadio et al., 2014, 2016, 2018b; Doppler et al., 2014, 2015, 2017; NavarroOtano et al., 2015; Zange et al., 2015; Melli et al., 2018), the sensitivity was $76 \%$ and the specificity was $100 \%$ in a pathological approach (Table 2). Thus, the presence of $\alpha$-syn in the skin tissue 
of PD patients has been widely demonstrated. The distribution of $\mathrm{p}-\alpha$-syn has been analyzed and compared between clinical variants of $\alpha$-synucleinopathy. Donadio et al. (2016, 2018b) compared positive rates of $\mathrm{p}-\alpha$-syn in cervical, thigh, and leg (malleolus) regions. In PD and DLB patients, all cervical regions were positive, but the positivity rate was decreased in the order of the thigh and leg. Conversely, in MSA patients, the leg had the highest positivity rate at about $70 \%$ ( $40 \%$ in $\mathrm{PD})$ and the positivity rate was decreased in the order of thigh and cervical regions, which was the opposite trend to PD and DLB. Patients with pure autonomic failure (PAF), which is a prodromal stage of synucleinopathies, had $100 \%$ positivity in all three sites. In a comparison of skin tissues from PD and DLB patients without $\mathrm{OH}$, the most sensitive site was the central region (especially the posterior cervical region). However, in cases with $\mathrm{OH}, \mathrm{p}-\alpha$-syn is widely distributed, and in the early stage of the disease, $p$ - $\alpha$-syn is detected first in the feet (Donadio et al., 2014, 2018a; Doppler et al., 2015; Niemann et al., 2021). These reports suggest that the site of the skin biopsy and the symptoms of patients have a significant effect on differential diagnosis of synucleinopathies.

\section{DETECTION OF AGGREGATED $\alpha$-SYNUCLEIN IN THE SKIN BY REAL-TIME QUAKING-INDUCED CONVERSION}

Manne et al. (2020) attempted to detect aggregated $\alpha$-syn by RTQuIC in frozen tissue and in formalin paraffin-embedded (FFPE) sections of occipital skin tissue from PD patients diagnosed by autopsy (Table 2). Frozen tissues from 24 of 25 PD patients and 1 of 25 healthy controls were positive in the RT-QuIC assay (sensitivity: 96\%; specificity: 96\%). In FFPE sections, 9 of 12 PD patients and 2 of 12 healthy controls were positive (sensitivity: $75 \%$; specificity: $83 \%$ ). The reasons for the low sensitivity and specificity in FFPE sections are thought to be that the amount of tissue used for the test was limited and the seeding activity was suppressed by formalin fixation.

Wang et al. (2021) analyzed abdominal skin of 130 cadavers with PD, DLB, MSA, AD, PSP, or CBD by the $\alpha$-syn RTQuIC assay. They detected aggregated $\alpha$-syn in 44 of $47 \mathrm{PD}$ samples, and all 7 DLB samples and 2 of 3 MSA samples were positive (sensitivities of 94,100 , and 67\%, respectively; Table 2; Wang et al., 2021). The combined sensitivity of the three $\alpha$-synucleinopathies was 93\%. The specificity for NNC, PSP, CBD, and $\mathrm{AD}$ combined was $93 \%$ and that for NNC alone was $98 \%$. The authors also used PMCA (substrate was mouse brain homogenate and induced conversion by sonication) (Nicot et al., 2019) to detect aggregated $\alpha$-syn in the same samples. The sensitivity and specificity to distinguish $\alpha$-synucleinopathies from non- $\alpha$-synucleinopathies were 82 and $96 \%$, respectively. The concordance rate for PD was 78.6\%, which was slightly higher than that using RT-QuIC, but there was no significant difference in the McNemar test. The authors noted that the accuracy of both detection methods was about the same. Additionally, the authors compared the sensitivity and specificity of these two methods for detection of aggregated $\alpha$-syn in the skin of living patients. The sensitivity and specificity of RT-QuIC were 95 and $100 \%$, respectively, whereas those of PMCA were 80 and $90 \%$, respectively, which is consistent with reports that the positive rate of aggregated $\alpha$-syn in the RT-QuIC assay is higher in skin samples near the center of the body than in other skin samples.

The sensitivity and specificity of $\alpha$-syn RT-QuIC using autopsy and biopsy samples are comparable with those using CSF, but the control group in the study using biopsy samples comprised healthy controls. Because all seven DLB autopsy cases were positive, it is possible that biopsies can also be used.

Wang et al. (2021) also performed RT-QuIC using leg lesions from skin biopsies of PD patients. In contrast to the cervical region, which showed an increase in ThT fluorescence within $30 \mathrm{~h}$, the leg skin tissue showed a weaker aggregation response and required more than $50 \mathrm{~h}$ to show a positive signal. This is the same result as detection of $p-\alpha$-syn by immunostaining. Thus, the skin collection site greatly affects the accuracy of diagnosis (Tsukita et al., 2019). These results led to the conclusion that examination of skin samples from appropriate sites may be helpful to differentiate diseases.

One study compared the accuracy of skin immunofluorescence (IF), skin RT-QuIC, and CSF RT-QuIC in diagnosing $\alpha$-synucleinopathies (Donadio et al., 2021). The sensitivity and specificity of skin IF were 90 and 100\%, those of skin RT-QuIC were 86 and 80\%, and those of CSF RT-QuIC were 78 and $100 \%$, respectively. These results suggest that skin IF is the most reliable. However, the sensitivity of skin IF in nine reports of skin IF analyzed by Tsukita et al. (2019) was low (76\%; Table 2). Moreover, the total sensitivity of CSF RT-QuIC for both PD and DLB as shown in Table 1 was $94 \%$ and the specificity for $\mathrm{AD}$ and healthy controls was $93 \%$. Therefore, the sensitivity of CSF RT-QuIC appears to be higher than that of skin IF. Currently, it is unclear which method is better, but it is important to at least combine these methods to take advantage of their characteristics.

\section{Detection of Aggregated $\alpha$-Synuclein in Cerebrospinal Fluid of Multiple System Atrophy Patients}

Detection of aggregated $\alpha$-syn in the CSF of MSA patients was conducted in two studies. The sensitivities were 6.5\% (Rossi et al., 2020) and 35\% (van Rumund et al., 2019), which are much lower than those for AD and DLB. Structural differences in the fibrils of aggregated $\alpha$-syn between MSA and PD have been suggested as the reason for the low sensitivity (Bousset et al., 2013; Peelaerts et al., 2015; Shahnawaz et al., 2020), which is similar to the differences in the biochemical properties of prion strains (Sano et al., 2015; Morales, 2017).

De Luca et al. (2019) attempted to detect aggregated $\alpha$ syn by RT-QuIC using OM of MSA patients. RT-QuIC using OM samples is also a successful method for prion detection (Bongianni et al., 2017). OM samples were collected from clinically diagnosed PD and MSA patients and RT-QuIC was performed. Ten of 18 (56\%) PD patients were positive, whereas 9 of 11 (82\%) MSA patients were positive. Additionally, 1 of 6 
TABLE 2 | Sensitivity and specificity of the detection of abnormal $\alpha$-synuclein aggregation in skin tissues.

\begin{tabular}{|c|c|c|c|c|c|c|c|c|c|c|}
\hline Method & Seed/antibody & Sample & $\alpha$-Synucleinopathy & Number & Sensitivity (\%) & $\begin{array}{l}\text { Non- } \alpha \\
\text {-synucleinopathy }\end{array}$ & Number & Specificity (\%) & & References \\
\hline \multirow[t]{8}{*}{ RT-QulC } & Homogenate & Autopsy & PD & 18 & 100 & Healthy control & 25 & 96.0 & & Manne et al., 2020 \\
\hline & FFPE & & PD & 28 & 92.9 & Healthy control & 12 & 83.3 & & \\
\hline & Emulgion (abdominal) & Autopsy & $\begin{array}{l}\text { Total } \\
\text { ( } \alpha \text {-synucleinopathies) }\end{array}$ & 57 & 93.0 & $\begin{array}{l}\text { Total (non- } \alpha- \\
\text { synucleinopathy) }\end{array}$ & 73 & 91.8 & & Wang et al., 2021 \\
\hline & & & PD & 47 & 93.6 & $\begin{array}{l}\text { Non-neurodegenerative } \\
\text { control }\end{array}$ & 43 & 97.7 & & \\
\hline & & & LBD & 7 & 100 & $A D$ & 17 & 70.6 & & \\
\hline & & & MSA & 3 & 66.7 & CBD & 5 & 100 & & \\
\hline & & & & & & PSP & 8 & 100 & & \\
\hline & Homogenate (posterior cervical) & Biopsy & PD & 20 & 95.0 & Non-PD & 21 & 100 & & \\
\hline \multirow[t]{2}{*}{ IF } & Anti-n- $\alpha$-syn antibody & Biopsy & PD & 38 & 76.3 & Healthy control & 42 & 59.5 & $\begin{array}{l}\text { Pooled } \\
\text { data of } \\
\text { three } \\
\text { studies }\end{array}$ & Tsukita et al., 2019 \\
\hline & Anti-p- $\alpha$-syn antibody & Biopsy & PD & 170 & 75.9 & Healthy control & 214 & 100 & $\begin{array}{l}\text { Pooled } \\
\text { data of nine } \\
\text { studies }\end{array}$ & \\
\hline
\end{tabular}

Anti-n- $\alpha$-syn antibody, anti-normal $\alpha$-syn antibody; Anti-p- $\alpha$-syn antibody, anti-phosphorylated $\alpha$-syn antibody; FFPE, formalin fixed paraffin embedded. 
and 2 of 12 patients were positive for CBD and PSP, respectively. RT-QuIC using OM samples has the potential to be a diagnostic tool for MSA, although large-scale studies are needed. The $\alpha$-synPMCA (1 min shake at $500 \mathrm{rpm}$ followed by $29 \mathrm{~min}$ rest) assay discriminates between CSF samples from PD and MSA patients with an overall sensitivity of $95.4 \%$. They used a combination of biochemical, biophysical, and biological methods to analyze the product of $\alpha$-syn-PMCA and found that the characteristics of the $\alpha$-syn aggregates in CSF can be used to readily distinguish between PD and MSA. They also found that the properties of aggregates amplified from CSF were similar to those from the brain. Furthermore, there are structural differences between $\alpha$ syn aggregates derived from patients with PD or MSA in Cryo-ET (Shahnawaz et al., 2020).

\section{DETECTION OF AGGREGATED $\alpha$-SYNUCLEIN IN PATIENT SAMPLES AT THE PRODROMAL STAGE OF $\alpha$-SYNUCLEINOPATHIES}

Idiopathic/isolated rapid eye movement (REM) sleep behavior disorder (iRBD) is a disorder characterized by behavioral abnormalities during REM sleep (Sateia, 2014; St Louis et al., 2017). PAF is a sporadic, slow progressive disorder that develops in adulthood and is clinically characterized by $\mathrm{OH}$ with a tendency to faint. The clinical features are $\mathrm{OH}$ with a tendency for syncope (No authors listed, 1996; Coon et al., 2019). These disorders are strong early signs of $\alpha$-synucleinopathies.

$\alpha$-Synuclein aggregates have been detected in CSF from 18 iRBD and 28 PAF patients with sensitivities of 100 and 92.9\%, respectively (Table 3; Rossi et al., 2020), whereas all $3 \mathrm{RBD}$ patients in the first report of $\alpha$-syn RT-QuIC were negative (Fairfoul et al., 2016). A large cohort study of iRBD patients has also been reported. $\alpha$-Syn RT-QuIC was performed using the CSF of 52 iRBD patients and 40 healthy controls to investigate whether aggregated $\alpha$-syn can be a biomarker for prodromal $\alpha$-synucleinopathy in a long-term follow-up after spinal fluid collection (Table 3; Iranzo et al., 2021). Forty-seven patients (90\%) in the iRBD group and four patients (10\%) in the healthy control group were positive. Thirty-two patients developed PD or DLB after a mean of 3.4 years, and among them, 31 patients (97\%) were positive in RT-QuIC (Table 3).

Detection in OM samples has also been reported. Twentyeight of 63 (44.4\%) iRBD patients and 19 of $41(46.3 \%)$ PD patients were positive (Stefani et al., 2021). The sensitivity in PD patients was similar to that reported by De Luca et al. (2019; Table 3). Although the sensitivity was not high, this result suggests that aggregated $\alpha$-syn is already present in $\mathrm{OM}$ at the iRBD stage. RT-QuIC using skin has not yet been reported, whereas p- $\alpha$-syn has been detected by IF in 23 of 28 iRBD patients without PD or DLB (Al-Qassabi et al., 2020). Therefore, RT-QuIC using skin tissues of $\mathrm{iRBD}$ patients may also be possible.

It has been reported that $>90 \%$ of patients develop $\alpha$ synucleinopathy within 14 years of diagnosis of iRBD (Iranzo et al., 2013, 2014) and $24-34 \%$ of PAF patients develop $\alpha$ synucleinopathies (Mabuchi et al., 2005; Kaufmann et al., 2017; Singer et al., 2017; Coon et al., 2020). It has been suggested that patients who are positive in RT-QuIC at the time of iRBD diagnosis have a high probability of developing $\alpha$-synucleinopathy. Therefore, the establishment of RT-QuIC for patients with iRBD and PAF may enable prediction of the onset of $\alpha$-synucleinopathies and therapeutic intervention before the onset.

\section{Differentiation of Patients With Rapidly Progressive Dementia}

Sano et al. (2018) performed $\alpha$-syn RT-QuIC on autopsied brain samples and found that all seven DLB cases were positive, whereas all CJD and $\mathrm{AD}$ cases were negative. RTQuIC has also been performed using CSF from 77 autopsied cases of suspected CJD, which showed positive results in all cases $(100 \%$ sensitivity) and 18 of 20 other neurodegenerative diseases with $\alpha$-synucleinopathy co-pathology were positive ( $90 \%$ sensitivity) (Bongianni et al., 2019). The combined sensitivity for all $\alpha$-synucleinopathies was $92.9 \%$. Additionally, only 2 of 49 neuropathological diagnoses of non- $\alpha$-synucleinopathies, which included 19 sporadic CJD, were positive, with a specificity of 95.9\% (Table 1). These reports indicate that $\alpha$-syn RT-QuIC can be used to accurately differentiate DLB from CJD-suspected cases (Rossi et al., 2020), but the number in this study and the standardization of $\alpha$-syn RT-QuIC were insufficient. We assumed

TABLE 3 | Sensitivity and specificity of RT-QulC in patients at the prodromal stage of $\alpha$-synucleinopathy.

\begin{tabular}{|c|c|c|c|c|c|c|c|}
\hline Substrate & $\alpha$-Synucleinopathy & Number & Sensitivity (\%) & Non- $\alpha$-synucleinopathy & Number & Specificity (\%) & References \\
\hline \multirow[t]{7}{*}{ CSF } & Risk for PD (RBD) & 3 & 0 & See Table 1 & & & Fairfoul et al., 2016 \\
\hline & iRBD & 18 & 100 & See Table 1 & & & Rossi et al., 2020 \\
\hline & PAF & 28 & 92.9 & & & & \\
\hline & Total iRBD & 52 & 90.4 & Total non-iRBD & 51 & 92.2 & Iranzo et al., 2021 \\
\hline & Disease free & 20 & 80.0 & Healthy control & 40 & 90.0 & \\
\hline & Converted to PD & 16 & 93.8 & Autosomal dominant AD & 5 & 100 & \\
\hline & Converted to DLB & 16 & 100 & Narcolepsy type 1 & 6 & 100 & \\
\hline \multirow[t]{2}{*}{$\mathrm{OM}$} & iRBD & 63 & 44.4 & Healthy control & 10 & 89.8 & Stefani et al., 2021 \\
\hline & PD & 41 & 46.3 & & & & \\
\hline
\end{tabular}

iRBD, idiopathic/isolated rapid eye movement (REM) sleep behavior disorder; PAF, pure autonomic failure. 
that $\alpha$-syn RT-QuIC was not capable of differentiating DLB from CJD in the last few years, but $\alpha$-syn RT-QuIC, prion-QuIC, and tau-QuIC assays can differentiate DLB from other rapidly progressive dementias (Sano et al., 2018).

\section{DISCUSSION}

\section{False Positive Is False?}

In many clinicopathological studies conducted to date, healthy controls, and AD, PSP, and CBD patients were included as negative control groups, which reported some "false positive" results. Cross-seeding of pathological proteins, such as tau and amyloid $\beta$, has been reported in vitro and in animal models, which acted as the seed for $\alpha$-syn (Clinton et al., 2010; Candelise et al., 2019). To resolve this problem, it is necessary to find a substrate or seed that does not cause cross-seeding. Mixed pathologies can be caused by cross-seeding. In a clinical study, it was reported that 88 of 147 patients diagnosed with $\mathrm{AD}$ were positive for $\alpha$-syn (Ronald, 2000). Fairfoul et al. (2016) reported a 65\% positive rate in patients with mixed $\mathrm{AD}$ and $\mathrm{LBD}$, and $15 \%$ in patients with $\mathrm{AD}$ and $\mathrm{LB}$. If such cases are clinically diagnosed as $\mathrm{AD}$, they may be the cause of false positives.

\section{THE LIMIT OF THE $\alpha$-SYNUCLEIN REAL-TIME QUAKING-INDUCED CONVERSION ASSAY}

Many researchers, ourselves included, are developing methods for the $\alpha$-syn RT-QuIC assay. Evidence is also being established to show that the $\alpha$-syn RT-QuIC method is particularly useful to diagnose DLB and MSA-P in $\alpha$-synucleinopathies. However, there are currently no biomarkers that reflect the clinical time course during clinical care. In particular, the development of synuclein PET is progressing, but there is still no ideal probe that can be advanced to clinical trials. Even if the development of $\alpha$-syn RT-QuIC progresses, $\alpha$-syn RT-QuIC has difficulty in reflecting chronological biomarkers. $\alpha$-Syn RT-QuIC is useful for diagnosis, but $\alpha$-syn RT-QuIC does have a biomarker that reflects chronological biomarkers. We believe that this is a limit of the $\alpha$-syn RT-QuIC assay.

\section{CONCLUSION}

Parkinson's disease and DLB can be diagnosed with high accuracy by $\alpha$-syn RT-QuIC, but the sensitivity and specificity

\section{REFERENCES}

Adler, C. H., Beach, T. G., Hentz, J. G., Shill, H. A., Caviness, J. N., Driver-Dunckley, E., et al. (2014). Low clinical diagnostic accuracy of early vs advanced Parkinson disease: clinicopathologic study. Neurology 83, 406-412. doi: 10.1212/WNL. 0000000000000641

Al-Qassabi, A., Tsao, T. S., Racolta, A., Kremer, T., Cañamero, M., Belousov, A., et al. (2020). Immunohistochemical Detection of Synuclein Pathology of $\alpha$-syn RT-QuIC may be inferior to that of prion RTQuIC. This may be related to LBs not having a high disease specificity compared with abnormal PrP deposition in human prion diseases. In some studies on $\alpha$-syn RTQuIC, patients who were diagnosed by clinical symptoms were tested. The accuracy of the clinical diagnosis of PD has been reported to be about $80 \%$ (Adler et al., 2014; Marsili et al., 2018). Studies on RT-QuIC with follow-up and autopsy are indispensable to improve accuracy, although they are difficult because the disease course of $\alpha$-synucleinopathies is longer than that of CJD.

To establish diagnosis by RT-QuIC, it is desirable to develop a method that does not cause cross-seeding, a detection method employing body fluids that can be collected easier (e.g., tears, sweat, and saliva), and a method to differentiate the type of $\alpha$-synucleinopathy.

\section{AUTHOR CONTRIBUTIONS}

KS supervised the study and wrote the manuscript. NN and TN collected information, wrote the manuscript, and participated in discussions. All authors contributed to the article and approved the submitted version.

\section{FUNDING}

This review was financially supported by grants for scientific research from the Ministry of Health, Labour and Welfare of Japan (KSat: No. 14507303), the Research Committee of Prion Disease and Slow Virus Infection, Research on Policy Planning and Evaluation for Rare and Intractable Diseases, Health and Labour Sciences Research Grants, the Research Committee of Surveillance and Infection Control of Prion Disease, the Ministry of Health, Labour and Welfare of Japan, the Japan Agency for Medical Research and Development (AMED) (Grant No. 18ek0109362h0001), Nakatani Foundation for Advancement of Measuring Technologies in Biomedical Engineering (2020K011), and The Mitsubishi Foundation/Research Grants in the Natural Sciences (202010022).

\section{ACKNOWLEDGMENTS}

We thank Michal Bell, Ph.D., and Mitchell Arico from Edanz (https://jp.edanz.com/ac) for editing a draft of this manuscript.

in Skin in Idiopathic Rapid Eye Movement Sleep Behavior Disorder and Parkinsonism. Mov. Disord. 36, 895-904. doi: 10.1002/mds.2 8399

Armstrong, M. J., Litvan, I., Lang, A. E., Bak, T. H., Bhatia, K. P., Borroni, B., et al. (2013). Criteria for the diagnosis of corticobasal degeneration. Neurology 80, 496-503. doi: 10.1212/WNL.0b013e31827f0fd1

Ascari, L. M., Rocha, S. C., Goncalves, P. B., Vieira, T., and Cordeiro, Y. (2020). Challenges and Advances in Antemortem Diagnosis of Human Transmissible 
Spongiform Encephalopathies. Front. Bioeng. Biotechnol. 8:585896. doi: 10. 3389/fbioe.2020.585896

Atarashi, R., Satoh, K., Sano, K., Fuse, T., Yamaguchi, N., Ishibashi, D., et al. (2011). Ultrasensitive human prion detection in cerebrospinal fluid by real-time quaking-induced conversion. Nat. Med. 17, 175-178. doi: 10.1038/nm.2294

Atarashi, R., Wilham, J. M., Christensen, L., Hughson, A. G., Moore, R. A., Johnson, L. M., et al. (2008). Simplified ultrasensitive prion detection by recombinant $\operatorname{PrP}$ conversion with shaking. Nat. Methods 5, 211-212. doi: 10.1038/nmeth0308211

Baba, M., Nakajo, S., Tu, P. H., Tomita, T., Nakaya, K., Lee, V. M., et al. (1998). Aggregation of alpha-synuclein in Lewy bodies of sporadic Parkinson's disease and dementia with Lewy bodies. Am. J. Pathol. 152, 879-884.

Beach, T. G., Adler, C. H., Sue, L. I., Vedders, L., Lue, L. F., White, C. L., et al. (2010). Multi-organ distribution of phosphorylated $\alpha$-synuclein histopathology in subjects with Lewy body disorders. Acta Neuropathol. 119, 689-702. doi: 10.1007/s00401-010-0664-3

Bertoncini, C. W., Jung, Y. S., Fernandez, C. O., Hoyer, W., Griesinger, C., Jovin, T. M., et al. (2005). Release of long-range tertiary interactions potentiates aggregation of natively unstructured alpha-synuclein. Proc. Natl. Acad. Sci. U. S. A. 102, 1430-1435. doi: 10.1073/pnas.0407146102

Bongianni, M., Ladogana, A., Capaldi, S., Klotz, S., Baiardi, S., Cagnin, A., et al. (2019). alpha-Synuclein RT-QuIC assay in cerebrospinal fluid of patients with dementia with Lewy bodies. Ann. Clin. Transl. Neurol. 6, 2120-2126. doi: 10. 1002/acn3.50897

Bongianni, M., Orru, C., Groveman, B. R., Sacchetto, L., Fiorini, M., Tonoli, G., et al. (2017). Diagnosis of Human Prion Disease Using Real-Time QuakingInduced Conversion Testing of Olfactory Mucosa and Cerebrospinal Fluid Samples. JAMA Neurol. 74, 155-162. doi: 10.1001/jamaneurol.2016.4614

Bousset, L., Pieri, L., Ruiz-Arlandis, G., Gath, J., Jensen, P. H., Habenstein, B., et al. (2013). Structural and functional characterization of two alpha-synuclein strains. Nat. Commun. 4:2575. doi: 10.1038/ncomms 3575

Breydo, L., Wu, J. W., and Uversky, V. N. (2012). Alpha-synuclein misfolding and Parkinson's disease. Biochim. Biophys. Acta 1822, 261-285. doi: 10.1016/ j.bbadis.2011.10.002

Candelise, N., Baiardi, S., Franceschini, A., Rossi, M., and Parchi, P. (2020). Towards an improved early diagnosis of neurodegenerative diseases: the emerging role of in vitro conversion assays for protein amyloids. Acta Neuropathol. Commun. 8, 1-16. doi: 10.1186/s40478-020-00990-x

Candelise, N., Schmitz, M., Llorens, F., Villar-Piqué, A., Cramm, M., Thom, T., et al. (2019). Seeding variability of different alpha synuclein strains in synucleinopathies. Ann. Neurol. 85, 691-703. doi: 10.1002/ana.25446

Castilla, J., Saa, P., Morales, R., Abid, K., Maundrell, K., and Soto, C. (2006). Protein misfolding cyclic amplification for diagnosis and prion propagation studies. Methods Enzymol. 412, 3-21. doi: 10.1016/S0076-6879(06)12001-7

Cheng, K., Wu, Q., Zhang, Z., Pielak, G. J., Liu, M., and Li, C. (2018). Crowding and Confinement Can Oppositely Affect Protein Stability. Chemphyschem 19, 3350-3355. doi: 10.1002/cphc.201800857

Clinton, L. K., Blurton-Jones, M., Myczek, K., Trojanowski, J. Q., and LaFerla, F. M. (2010). Synergistic interactions between $A \beta$, tau, and $\alpha$-synuclein: acceleration of neuropathology and cognitive decline. J. Neurosci. 30, 7281-7289. doi: 10. 1523/JNEUROSCI.0490- 10.2010

Coon, E. A., Mandrekar, J. N., Berini, S. E., Benarroch, E. E., Sandroni, P., Low, P. A., et al. (2020). Predicting phenoconversion in pure autonomic failure. Neurology 95, e889-e897. doi: 10.1212/WNL.0000000000010002

Coon, E. A., Singer, W., and Low, P. A. (2019). Pure Autonomic Failure. Mayo Clin. Proc. 94, 2087-2098. doi: 10.1016/j.mayocp.2019.03.009

Crowther, R. A., Jakes, R., Spillantini, M. G., and Goedert, M. (1998). Synthetic filaments assembled from C-terminally truncated alpha-synuclein. FEBS Lett. 436, 309-312. doi: 10.1016/s0014-5793(98)01146-6

De Luca, C. M. G., Elia, A. E., Portaleone, S. M., Cazzaniga, F. A., Rossi, M., Bistaffa, E., et al. (2019). Efficient RT-QuIC seeding activity for $\alpha$-synuclein in olfactory mucosa samples of patients with Parkinson's disease and multiple system atrophy. Transl. Neurodegener. 8, 1-14. doi: 10.1186/s40035-0190164-x

de Oliveira, G. A. P., Cordeiro, Y., Silva, J. L., and Vieira, T. (2019). Liquidliquid phase transitions and amyloid aggregation in proteins related to cancer and neurodegenerative diseases. Adv. Protein Chem. Struct. Biol. 118, 289-331. doi: 10.1016/bs.apcsb.2019.08.002
Donadio, V., Incensi, A., El-Agnaf, O., Rizzo, G., Vaikath, N., Del Sorbo, F., et al. (2018b). Skin alpha-synuclein deposits differ in clinical variants of synucleinopathy: an in vivo study. Sci. Rep. 8:14246. doi: 10.1038/s41598-01832588-8

Donadio, V., Incensi, A., Del Sorbo, F., Rizzo, G., Infante, R., Scaglione, C., et al. (2018a). Skin nerve phosphorylated a-synuclein deposits in Parkinson disease with orthostatic hypotension. J. Neuropathol. Exp. Neurol. 77, 942-949. doi: 10.1093/jnen/nly074

Donadio, V., Incensi, A., Leta, V., Giannoccaro, M. P., Scaglione, C., Martinelli, P., et al. (2014). Skin nerve alpha-synuclein deposits: a biomarker for idiopathic Parkinson disease. Neurology 82, 1362-1369. doi: 10.1212/WNL. 0000000000000316

Donadio, V., Incensi, A., Piccinini, C., Cortelli, P., Giannoccaro, M. P., Baruzzi, A., et al. (2016). Skin nerve misfolded $\alpha$-synuclein in pure autonomic failure and Parkinson disease. Ann. Neurol. 79, 306-316. doi: 10.1002/ana.24567

Donadio, V., Wang, Z., Incensi, A., Rizzo, G., Fileccia, E., Vacchiano, V., et al. (2021). In Vivo Diagnosis of Synucleinopathies: a Comparative Study of Skin Biopsy and RT-QuIC. Neurology 96:10.1212/WNL.0000000000011935. doi: 10. 1212/WNL.0000000000011935

Donaghy, P. C., and McKeith, I. G. (2014). The clinical characteristics of dementia with Lewy bodies and a consideration of prodromal diagnosis. Alzheimers Res. Ther. 6:46. doi: 10.1186/alzrt274

Doppler, K., Ebert, S., Uceyler, N., Trenkwalder, C., Ebentheuer, J., Volkmann, J., et al. (2014). Cutaneous neuropathy in Parkinson's disease: a window into brain pathology. Acta Neuropathol. 128, 99-109. doi: 10.1007/s00401-014-1284-0

Doppler, K., Jentschke, H. M., Schulmeyer, L., Vadasz, D., Janzen, A., Luster, M., et al. (2017). Dermal phospho-alpha-synuclein deposits confirm REM sleep behaviour disorder as prodromal Parkinson's disease. Acta Neuropathol. 133, 535-545. doi: 10.1007/s00401-017-1684-z

Doppler, K., Weis, J., Karl, K., Ebert, S., Ebentheuer, J., Trenkwalder, C., et al. (2015). Distinctive distribution of phospho-alpha-synuclein in dermal nerves in multiple system atrophy. Mov. Disord. 30, 1688-1692. doi: 10.1002/mds.26293

Fabbrini, G., Fabbrini, A., and Suppa, A. (2019). Progressive supranuclear palsy, multiple system atrophy and corticobasal degeneration. Handb. Clin. Neurol. 165, 155-177. doi: 10.1016/B978-0-444-64012-3.00009-5

Fairfoul, G., McGuire, L. I., Pal, S., Ironside, J. W., Neumann, J., Christie, S., et al. (2016). Alpha-synuclein RT-QuIC in the CSF of patients with alphasynucleinopathies. Ann. Clin. Transl. Neurol. 3, 812-818. doi: 10.1002/acn3. 338

Foutz, A., Appleby, B. S., Hamlin, C., Liu, X., Yang, S., Cohen, Y., et al. (2017). Diagnostic and prognostic value of human prion detection in cerebrospinal fluid. Ann. Neurol. 81, 79-92. doi: 10.1002/ana.24833

Fujiwara, H., Hasegawa, M., Dohmae, N., Kawashima, A., Masliah, E., Goldberg, M. S., et al. (2002). alpha-Synuclein is phosphorylated in synucleinopathy lesions. Nat. Cell Biol. 4, 160-164. doi: 10.1038/ncb748

Garrido, A., Fairfoul, G., Tolosa, E. S., Martí, M. J., and Green, A. (2019). $\alpha-$ synuclein RT-QuIC in cerebrospinal fluid of LRRK2-linked Parkinson's disease. Ann. Clin. Transl. Neurol. 6, 1024-1032. doi: 10.1002/acn3.772

Geschwind, M. D., and Murray, K. (2018). Differential diagnosis with other rapid progressive dementias in human prion diseases. Handb. Clin. Neurol. 153, 371-397. doi: 10.1016/B978-0-444-63945-5.00020-9

Gibbons, C. H., Garcia, J., Wang, N., Shih, L. C., and Freeman, R. (2016). The diagnostic discrimination of cutaneous $\alpha$-synuclein deposition in Parkinson disease. Neurology 87, 505-512.

Gilman, S., Wenning, G. K., Low, P. A., Brooks, D. J., Mathias, C. J., Trojanowski, J. Q., et al. (2008). Second consensus statement on the diagnosis of multiple system atrophy. Neurology 71, 670-676. doi: 10.1212/01.wnl.0000324625.004 04.15

Gnutt, D., and Ebbinghaus, S. (2016). The macromolecular crowding effect-from in vitro into the cell. Biol. Chem. 397, 37-44. doi: 10.1515/hsz-2015-0161

Groveman, B. R., Orrù, C. D., Hughson, A. G., Raymond, L. D., Zanusso, G., Ghetti, B., et al. (2018). Rapid and ultra-sensitive quantitation of disease-associated $\alpha$-synuclein seeds in brain and cerebrospinal fluid by $\alpha$ Syn RT-QuIC. Acta Neuropathol. Commun. 6, 1-10. doi: 10.1186/s40478-018-0508-2

Healy, D. G., Falchi, M., O'Sullivan, S. S., Bonifati, V., Durr, A., Bressman, S., et al. (2008). Phenotype, genotype, and worldwide genetic penetrance of LRRK2associated Parkinson's disease: a case-control study. Lancet Neurol. 7, 583-590. doi: 10.1016/S1474-4422(08)70117-0 
Hermann, P., Appleby, B., Brandel, J. P., Caughey, B., Collins, S., Geschwind, M. D., et al. (2021). Biomarkers and diagnostic guidelines for sporadic CreutzfeldtJakob disease. Lancet Neurol. 20, 235-246. doi: 10.1016/S1474-4422(20)30 $477-4$

Hoglinger, G. U., Respondek, G., Stamelou, M., Kurz, C., Josephs, K. A., Lang, A. E., et al. (2017). Clinical diagnosis of progressive supranuclear palsy: the movement disorder society criteria. Mov. Disord. 32, 853-864. doi: 10.1002/mds. 26987

Hughes, A. J., Daniel, S. E., Kilford, L., and Lees, A. J. (1992). Accuracy of clinical diagnosis of idiopathic Parkinson's disease: a clinico-pathological study of 100 cases. J. Neurol. Neurosurg. Psychiatry 55, 181-184. doi: 10.1136/jnnp.55.3.181

Ikemura, M., Saito, Y., Sengoku, R., Sakiyama, Y., Hatsuta, H., Kanemaru, K., et al. (2008). "Lewy Body Pathology Involves Cutaneous Nerves. J. Neuropathol. Exp. Neurol. 67, 945-953.

Iranzo, A., Fairfoul, G., Ayudhaya, A. C. N., Serradell, M., Gelpi, E., Vilaseca, I., et al. (2021). Detection of alpha-synuclein in CSF by RT-QuIC in patients with isolated rapid-eye-movement sleep behaviour disorder: a longitudinal observational study. Lancet Neurol. 20, 203-212. doi: 10.1016/S1474-4422(20) 30449-X

Iranzo, A., Fernández-Arcos, A., Tolosa, E., Serradell, M., Molinuevo, J. L., Valldeoriola, F., et al. (2014). Neurodegenerative disorder risk in idiopathic REM sleep behavior disorder: study in 174 patients. PLoS One 9:e89741. doi: 10.1371/journal.pone.0089741

Iranzo, A., Tolosa, E., Gelpi, E., Molinuevo, J. L., Valldeoriola, F., Serradell, M., et al. (2013). Neurodegenerative disease status and post-mortem pathology in idiopathic rapid-eye-movement sleep behaviour disorder: an observational cohort study. Lancet Neurol. 12, 443-453. doi: 10.1016/S1474-4422(13)70056-5

Kaji, S., Maki, T., Ishimoto, T., Yamakado, H., and Takahashi, R. (2020). Insights into the pathogenesis of multiple system atrophy: focus on glial cytoplasmic inclusions. Transl. Neurodegener. 9:7. doi: 10.1186/s40035-020-0185-5

Kang, U. J., Boehme, A. K., Fairfoul, G., Shahnawaz, M., Ma, T. C., Hutten, S. J., et al. (2019). Comparative study of cerebrospinal fluid $\alpha$-synuclein seeding aggregation assays for diagnosis of Parkinson's disease. Mov. Disord. 34, 536544. doi: $10.1002 / \mathrm{mds} .27646$

Kaufmann, H., Norcliffe-Kaufmann, L., Palma, J. A., Biaggioni, I., Low, P. A., Singer, W., et al. (2017). Natural history of pure autonomic failure: a United States prospective cohort. Ann. Neurol. 81, 287-297. doi: 10.1002/ana. 24877

Kosaka, K. (1978). Lewy bodies in cerebral cortex, report of three cases. Acta Neuropathol. 42, 127-134. doi: 10.1007/BF00690978

Lee, J. M., Derkinderen, P., Kordower, J. H., Freeman, R., Munoz, D. G., Kremer, T., et al. (2017). The search for a peripheral biopsy indicator of $\alpha$-synuclein pathology for Parkinson disease. J. Neuropathol. Exp. Neurol. 76, 2-15. doi: 10.1093/jnen/nlw103

Lucetti, C., Logi, C., Del Dotto, P., Berti, C., Ceravolo, R., Baldacci, F., et al. (2010). Levodopa response in dementia with lewy bodies: a 1-year follow-up study. Park. Relat. Disord. 16, 522-526. doi: 10.1016/j.parkreldis.2010.06.004

Mabuchi, N., Hirayama, M., Koike, Y., Watanabe, H., Ito, H., Kobayashi, R., et al. (2005). Progression and prognosis in pure autonomic failure (PAF): comparison with multiple system atrophy. J. Neurol. Neurosurg. Psychiatry 76, 947-952. doi: 10.1136/jnnp.2004.049023

Mancini, A., Mazzocchetti, P., Sciaccaluga, M., Megaro, A., Bellingacci, L., Beccano-Kelly, D. A., et al. (2020). From Synaptic Dysfunction to Neuroprotective Strategies in Genetic Parkinson's Disease: lessons From LRRK2. Front. Cell Neurosci. 14:158. doi: 10.3389/fncel.2020.00158

Manne, S., Kondru, N., Hepker, M., Jin, H., Anantharam, V., Lewis, M., et al. (2019). Ultrasensitive Detection of Aggregated $\alpha$-Synuclein in Glial Cells, Human Cerebrospinal Fluid, and Brain Tissue Using the RT-QuIC Assay: new High-Throughput Neuroimmune Biomarker Assay for Parkinsonian Disorders. J. Neuroimmune Pharmacol. 14, 423-435. doi: 10.1007/s11481-01909835-4

Manne, S., Kondru, N., Jin, H., Serrano, G. E., Anantharam, V., Kanthasamy, A., et al. (2020). Blinded RT-QuIC Analysis of $\alpha$-Synuclein Biomarker in Skin Tissue From Parkinson's Disease Patients. Mov. Disord. 35, 2230-2239. doi: $10.1002 / \mathrm{mds} .28242$

Marsili, L., Rizzo, G., and Colosimo, C. (2018). Diagnostic Criteria for Parkinson's Disease: from James Parkinson to the Concept of Prodromal Disease. Front. Neurol. 9:156. doi: 10.3389/fneur.2018.00156
McKeith, I. G., Boeve, B. F., Dickson, D. W., Halliday, G., Taylor, J. P., Weintraub, D., et al. (2017). Diagnosis and management of dementia with Lewy bodies: fourth consensus report of the DLB Consortium. Neurology 89, 88-100. doi: 10.1212/WNL.0000000000004058

Melli, G., Vacchi, E., Biemmi, V., Galati, S., Staedler, C., Ambrosini, R., et al. (2018). Cervical skin denervation associates with alpha-synuclein aggregates in Parkinson disease. Ann. Clin. Transl. Neurol. 5, 1394-1407. doi: 10.1002/acn3. 669

Mitsui, J., Matsukawa, T., Sasaki, H., Yabe, I., Matsushima, M., Durr, A., et al. (2015). Variants associated with Gaucher disease in multiple system atrophy. Ann. Clin. Transl. Neurol. 2, 417-426. doi: 10.1002/acn3.185

Morales, R. (2017). Prion strains in mammals: different conformations leading to disease. PLoS Pathog. 13:e1006323. doi: 10.1371/journal.ppat.1006323

Murray, D. T., Kato, M., Lin, Y., Thurber, K. R., Hung, I., McKnight, S. L., et al. (2017). Structure of FUS Protein Fibrils and Its Relevance to Self-Assembly and Phase Separation of Low-Complexity Domains. Cell 171, 615-627.e16. doi: 10.1016/j.cell.2017.08.048

Nakazato, Y., Yamazaki, H., Hirato, J., Ishida, Y., and Yamaguchi, H. (1990). Oligodendroglial microtubular tangles in olivopontocerebellar atrophy. J. Neuropathol. Exp. Neurol. 49, 521-530. doi: 10.1097/00005072-19900900000007

Navarro-Otano, J., Casanova-Molla, J., Morales, M., Valls-Sole, J., and Tolosa, E. (2015). Cutaneous autonomic denervation in Parkinson's disease. J. Neural. Transm. 122, 1149-1155. doi: 10.1007/s00702-014-1355-3

Nicot, S., Verchère, J., Bélondrade, M., Mayran, C., Bétemps, D., Bougard, D., et al. (2019). Seeded propagation of $\alpha$-synuclein aggregation in mouse brain using protein misfolding cyclic amplification. FASEB J. 33, 12073-12086. doi: 10.1096/fj.201900354R

Niemann, N., Billnitzer, A., and Jankovic, J. (2021). Parkinson's disease and skin. Park. Relat. Disord. 82, 61-76. doi: 10.1016/j.parkreldis.2020.11.017

No authors listed (1996). Consensus statement on the definition of orthostatic hypotension, pure autonomic failure, and multiple system atrophy. J. Neurol. Sci. 144, 218-219.

Orru, C. D., Bongianni, M., Tonoli, G., Ferrari, S., Hughson, A. G., Groveman, B. R., et al. (2014). A test for Creutzfeldt-Jakob disease using nasal brushings. N. Engl. J. Med. 371, 519-529. doi: 10.1056/NEJMoa1315200

Orrù, C. D., Ma, T. C., Hughson, A. G., Groveman, B. R., Srivastava, A., Galasko, D., et al. (2020). A rapid $\alpha$-synuclein seed assay of Parkinson's disease CSF panel shows high diagnostic accuracy. Ann. Clin. Transl. Neurol. 8, 374-384. doi: 10.1002/acn3.51280

Orru, C. D., Yuan, J., Appleby, B. S., Li, B., Li, Y., Winner, D., et al. (2017). Prion seeding activity and infectivity in skin samples from patients with sporadic Creutzfeldt-Jakob disease. Sci. Transl. Med. 9:eaam7785. doi: 10.1126/ scitranslmed.aam7785

Papp, M. I., Kahn, J. E., and Lantos, P. L. (1989). Glial cytoplasmic inclusions in the CNS of patients with multiple system atrophy (striatonigral degeneration, olivopontocerebellar atrophy and Shy-Drager syndrome). J. Neurol. Sci. 94, 79-100. doi: 10.1016/0022-510x(89)90219-0

Peelaerts, W., Bousset, L., Van der Perren, A., Moskalyuk, A., Pulizzi, R., Giugliano, M., et al. (2015). alpha-Synuclein strains cause distinct synucleinopathies after local and systemic administration. Nature 522, 340-344. doi: 10.1038/ nature 14547

Ray, S., Singh, N., Kumar, R., Patel, K., Pandey, S., Datta, D., et al. (2020). alphaSynuclein aggregation nucleates through liquid-liquid phase separation. Nat. Chem. 12, 705-716. doi: 10.1038/s41557-020-0465-9

Rivas, G., and Minton, A. P. (2016). Macromolecular Crowding In Vitro, In Vivo, and In Between. Trends Biochem. Sci. 41, 970-981. doi: 10.1016/j.tibs.2016.08. 013

Ronald, L. H. (2000). Lewy bodies in Alzheimer's disease a neuropathological review of 145 cases using alpha-synuclein immunohistochemistry. Brain Pathol. $10,378-384$.

Rossi, M., Candelise, N., Baiardi, S., Capellari, S., Giannini, G., Orrù, C. D., et al. (2020). Ultrasensitive RT-QuIC assay with high sensitivity and specificity for Lewy body-associated synucleinopathies. Acta Neuropathol. 140, 49-62. doi: 10.1007/s00401-020-02160-8

Saborio, G. P., Permanne, B., and Soto, C. (2001). Sensitive detection of pathological prion protein by cyclic amplification of protein misfolding. Nature 411, 810-813. doi: 10.1038/35081095 
Sano, K., Atarashi, R., and Nishida, N. (2015). Structural conservation of prion strain specificities in recombinant prion protein fibrils in real-time quakinginduced conversion. Prion 9, 237-243. doi: 10.1080/19336896.2015.1062201

Sano, K., Atarashi, R., Satoh, K., Ishibashi, D., Nakagaki, T., Iwasaki, Y., et al. (2018). Prion-Like Seeding of Misfolded $\alpha$-Synuclein in the Brains of Dementia with Lewy Body Patients in RT-QUIC. Mol. Neurobiol. 55, 3916-3930. doi: 10.1007/s12035-017-0624-1

Sano, K., Satoh, K., Atarashi, R., Takashima, H., Iwasaki, Y., Yoshida, M., et al. (2013). Early detection of abnormal prion protein in genetic human prion diseases now possible using real-time QUIC assay. PLoS One 8:e54915. doi: 10.1371/journal.pone.0054915

Sateia, M. J. (2014). International classification of sleep disorders-third edition: highlights and modifications. Chest 146, 1387-1394. doi: 10.1378/chest.140970

Satoh, K., Atarashi, R., and Nishida, N. (2017). Real-Time Quaking-Induced Conversion for Diagnosis of Prion Disease. Methods Mol. Biol. 1658, 305-310. doi: 10.1007/978-1-4939-7244-9_21

Satoh, K., Fuse, T., Nonaka, T., Dong, T., Takao, M., Nakagaki, T., et al. (2019). Postmortem Quantitative Analysis of Prion Seeding Activity in the Digestive System. Molecules 24:4601. doi: 10.3390/molecules24244601

Shahnawaz, M., Mukherjee, A., Pritzkow, S., Mendez, N., Rabadia, P., Liu, X., et al. (2020). Discriminating $\alpha$-synuclein strains in Parkinson's disease and multiple system atrophy. Nature 578, 273-277. doi: 10.1038/s41586-020-1984-7

Singer, W., Berini, S. E., Sandroni, P., Fealey, R. D., Coon, E. A., Suarez, M. D., et al. (2017). Pure autonomic failure: predictors of conversion to clinical CNS involvement. Neurology 88, 1129-1136. doi: 10.1212/WNL.0000000000003737

Spillantini, M. G., Schmidt, M. L., Lee, V. M., Trojanowski, J. Q., Jakes, R., and Goedert, M. (1997). Alpha-synuclein in Lewy bodies. Nature 388, 839-840. doi: $10.1038 / 42166$

St Louis, E. K., Boeve, A. R., and Boeve, B. F. (2017). REM sleep behavior disorder in Parkinson's disease and other synucleinopathies. Mov. Disord. 32, 645-658. doi: $10.1002 / \mathrm{mds} .27018$

Stefani, A., Iranzo, A., Holzknecht, E., Perra, D., Bongianni, M., Gaig, C., et al. (2021). Alpha-synuclein seeds in olfactory mucosa of patients with isolated REM sleep behaviour disorder. Brain 144, 1118-1126. doi: 10.1093/brain/ awab005

Stokholm, M. G., Danielsen, E. H., Hamilton-Dutoit, S. J., and Borghammer, P. (2016). Pathological alpha-synuclein in gastrointestinal tissues from prodromal Parkinson disease patients. Ann. Neurol. 79, 940-949. doi: 10.1002/ana.24648

Takatsuki, H., Fuse, T., Nakagaki, T., Mori, T., Mihara, B., Takao, M., et al. (2016). Prion-Seeding Activity Is widely Distributed in Tissues of Sporadic CreutzfeldtJakob Disease Patients. EBioMedicine 12, 150-155. doi: 10.1016/j.ebiom.2016. 08.033

Tange, H., Ishibashi, D., Nakagaki, T., Taguchi, Y., Kamatari, Y. O., Ozawa, H., et al. (2021). Liquid-liquid phase separation of full-length prion protein initiates conformational conversion in vitro. J. Biol. Chem. 296:100367. doi: 10.1016/j. jbc. 2021.100367

Tsukita, K., Sakamaki-Tsukita, H., Tanaka, K., Suenaga, T., and Takahashi, R. (2019). Value of in vivo $\alpha$-synuclein deposits in Parkinson's disease: a systematic review and meta-analysis. Mov. Disord. 34, 1452-1463.
Tysnes, O. B., and Storstein, A. (2017). Epidemiology of Parkinson's disease. J. Neural. Transm. 124, 901-905. doi: 10.1007/s00702-017-1 686-y

van Rumund, A., Green, A. J. E., Fairfoul, G., Esselink, R. A. J., Bloem, B. R., and Verbeek, M. M. (2019). $\alpha$-Synuclein real-time quaking-induced conversion in the cerebrospinal fluid of uncertain cases of parkinsonism. Ann. Neurol. 85, 777-781. doi: 10.1002/ana.25447

Villar-Piqué, A., Lopes da Fonseca, T., and Outeiro, T. F. (2016). Structure, function and toxicity of alpha-synuclein: the Bermuda triangle in synucleinopathies. J. Neurochem. 139, 240-255. doi: 10.1111/jnc.13249

Wakabayashi, K., Yoshimoto, M., Tsuji, S., and Takahashi, H. (1998). Alphasynuclein immunoreactivity in glial cytoplasmic inclusions in multiple system atrophy. Neurosci. Lett. 249, 180-182. doi: 10.1016/s0304-3940(98)00 407-8

Wang, C., Zhao, C., Li, D., Tian, Z., Lai, Y., Diao, J., et al. (2016). Versatile structures of $\alpha$-synuclein. Front. Mol. Neurosci. 9:48. doi: 10.3389/fnmol.2016.00048

Wang, N., Gibbons, C. H., Lafo, J., and Freeman, R. (2013). $\alpha$-Synuclein in cutaneous autonomic nerves. Neurlogy 81, 1604-1610. doi: 10.1212/WNL. 0b013e3182a9f449

Wang, Z., Becker, K., Donadio, V., Siedlak, S., Yuan, J., Rezaee, M., et al. (2021). Skin $\alpha$-Synuclein Aggregation Seeding Activity as a Novel Biomarker for Parkinson Disease. JAMA Neurol. 78, 1-11. doi: 10.1001/jamaneurol.2020. 3311

Wilham, J. M., Orru, C. D., Bessen, R. A., Atarashi, R., Sano, K., Race, B., et al. (2010). Rapid end-point quantitation of prion seeding activity with sensitivity comparable to bioassays. PLoS Pathog. 6:e1001217. doi: 10.1371/journal.ppat. 1001217

Zange, L., Noack, C., Hahn, K., Stenzel, W., and Lipp, A. (2015). Phosphorylated alpha-synuclein in skin nerve fibres differentiates Parkinson's disease from multiple system atrophy. Brain 138, 2310-2321. doi: 10.1093/brain/awv138

Zerr, I., and Hermann, P. (2018). Diagnostic challenges in rapidly progressive dementia. Expert Rev. Neurother. 18, 761-772. doi: 10.1080/14737175.2018. 1519397

Conflict of Interest: The authors declare that the research was conducted in the absence of any commercial or financial relationships that could be construed as a potential conflict of interest.

Publisher's Note: All claims expressed in this article are solely those of the authors and do not necessarily represent those of their affiliated organizations, or those of the publisher, the editors and the reviewers. Any product that may be evaluated in this article, or claim that may be made by its manufacturer, is not guaranteed or endorsed by the publisher.

Copyright (c) 2021 Nakagaki, Nishida and Satoh. This is an open-access article distributed under the terms of the Creative Commons Attribution License (CC BY). The use, distribution or reproduction in other forums is permitted, provided the original author(s) and the copyright owner(s) are credited and that the original publication in this journal is cited, in accordance with accepted academic practice. No use, distribution or reproduction is permitted which does not comply with these terms. 